\title{
ISOLATED CYSTICERCOSIS OF ANTERIOR ABDOMINAL WALL MIMICKING CLINICALLY AS ACUTE APPENDICITIS: AN UNUSUAL PRESENTATION
}

\author{
Gupta M.K. ${ }^{1}$, Ahmad K. ${ }^{2}$, Ansari S. ${ }^{3}$, Rauniyar R.K. ${ }^{4}$, Chaudhary S. ${ }^{5}$
}

\begin{abstract}
Cysticercosis, a parasitic disease caused by Taenia solium larva, is a major public health problem in developing countries. The encysted larval stage can affect any part of the body, but are most frequently detected in brain, eye, skeletal muscle and subcutaneous tissues. Isolated cysticercosis of the abdominal wall can rarely mimic acute abdomen due to its nonspecific clinical presentation. We report a case of isolated cysticercosis of the right lower abdominal wall ina 25-year-old female who presented with acute abdominal pain mimicking clinically as acute appendicitis. High resolution ultrasonography showed typical features of cysticercosis in the abdominal wall and the patient had excellent clinical response to albendazole.
\end{abstract}

KEYWORDS: Acute appendicitis, Anterior abdominal wall, Cysticercosis, Ultrasonography.

1, 2, 3 \& 4. Department of Radiodiagnosis and Imaging, B. P. Koirala Institute of Health Sciences, Dharan, Nepal 5. Associate Professor, Department of Internal Medicine, Universal College of Medical Sciences \& Teaching Hospital, Bhairahawa, Nepal

\author{
For Correspondence \\ Dr. Mukesh Kumar Gupta, \\ Associate Professor, \\ Department of Radiodiagnosis and Imaging, \\ B.P. Koirala Institute of Health Sciences, Dharan, Nepal \\ Email: mukeshgupta148@yahoo.com
}




\section{INTRODUCTION}

Cysticercosis is a parasitic disease endemic in developing countries and its magnitude has increased in developed countries due to population mobility. ${ }^{1,2}$ Cysticercosis can affect various organs including the brain, spinalcord, orbit, muscle, subcutaneous tissue, and heart.The clinical manifestations of the patients vary depending on the site of larval encystment, number of the cyst and the extent of associated inflammatory responses or scarring. ${ }^{3,4}$ Soft tissue cysticercosis frequently presents with multiple lesions and involvement of central nervous system. Isolated involvement of soft tissue is rare and can mimic other soft tissue lesions including neoplasms and other infective or inflammatory pathologies. ${ }^{5}$ Radiological imaging plays an important role in establishing the diagnosis by demonstrating scolex on CT, MRI and/or ultrasonography (USG). The aim of our case report is to present an isolated case of cysticercosis of the anterior abdominal wall in right lower quadrant mimicking clinically as acute appendicitis.

\section{CASE REPORT}

A 25 year old female presented with sudden onset of acute abdominal pain in right lower quadrant that woke her up from sleep in the morning. There was no history of nausea, vomiting, loose motion, fever or trauma. Physical examination revealed tenderness over McBurney's point. A complete CNS and ocular examination were unremarkable. Laboratory investigation showed normal white blood count and ESR. She was clinically suspected to be a case of acute appendicitis and was referred for ultrasonography. High resolution ultrasound (figure1) revealed a well-defined cystic lesion of size $11 \times 9$ $\mathrm{mm}$ with an echogenic mural nodule (i.e. scolex) projecting into the cyst with associated pericystic inflammation in the muscle of anterior abdominal wall in right lower quadrant in the region of McBurney's point.

Figure 1: High resolution ultrasonography shows a welldefined cystic lesion with an echogenic mural nodule (i.e. scolex) projecting into the cyst with associated pericystic inflammation in the muscle of anterior abdominal wall in right lower quadrant suggestive of cysticercosis.

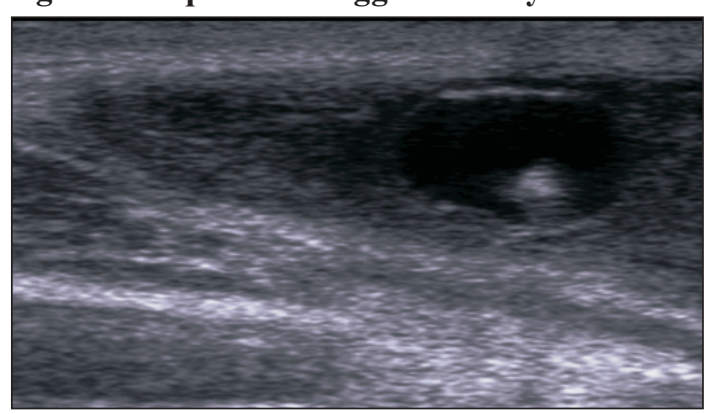

There was no any evidence of appendicitis on ultrasonography. Since the sonographic features were typical of muscle cysticercosis, FNAC or biopsy was not performed and empirical treatment with albendazole along with steroid was started. The patient had excellent clinical response and followup ultrasound at three months showed complete resolution of the cyst and edema.

\section{DISCUSSION}

Human cysticercosis is caused by infestation of encysted larvae of the tapewormTaenia solium, which results from consumption of food or water contaminated with viable eggs of Taenia solium or regurgitation of proglottids into the stomach from the intestine of people harboring a gravid worm. ${ }^{6}$ Cysticerci may be found in any organ, but it has strong predilection to involve central nervous system. Muscle infestation is usually associated with CNS involvement or with multiple cysts or both. The clinical presentation of cysticercosis depends primarily on the anatomic location, number of the cyst and extent of associated inflammatory response by the host. ${ }^{5,7}$ Clinically solitary soft tissue cysticercosis can be confused with soft tissue tumor and other infective or inflammatory pathologies. Moreover, abdominal wall involvement can mimic hernia or grave intrabdominal pathology including acute abdomen resulting in diagnostic dilemmas. Our patient presented with acute abdominal pain in right lower quadrant mimicking clinically as acute appendicitis because of the location of the cyst in the abdominal wall in the region of McBurney's point.

Serological test for specific anticysticercal antibodies has low sensitivity when the parasite burden is low as in solitary lesions. FNAC or excisional biopsy provides most definitive diagnosis by identification of scolex or the parasitic fragments. ${ }^{8}$ Radiological modalities like CT, MRI and USG play crucial role in establishing the diagnosis of cysticercosis. High frequency USG is readily available, cheaper and reliable modality for the diagnosis of soft tissue cysticercosis. Four different types of sonographic appearances of cysticercosis have been described by Vijayaraghavan. ${ }^{9}$ First type is cysticercus cyst containing echogenic scolex with inflammation around it, as a result of death of the larva. Similar picture was observed in our case. The second type is an irregular cyst with very minimal fluid on one side, indicating a leakage of fluid. The scolex is not seen within the cyst. It may be due to escape of the scolex outside the cyst or partial collapse of the cyst. The third appearance is a large irregular collection of exudative fluid within the muscle with the typical cysticercus cyst containing the scolex, situated eccentrically within the collection. This may be due to chronic intermittent 
leakage of fluid from the cyst, leading to florid inflammatory exudates. This appearance is similar to an intramuscular abscess, but the visualization of the cysticercus cyst within it clinches the diagnosis. The fourth appearance is of calcified cyst appearing as multiple elliptical calcifications in soft tissue.

Treatment of cysticercosis depends on the site of involvement, number of cyst and symptoms of the patient. Isolated soft tissue cysticercosis can be treated with surgical excision and/ or with antihelminthic medications such as albendazole or praziquantel in conjunction with steroids. ${ }^{6,10}$ Our patient was treated with oral albendazole and steroid and showed excellent response.

\section{CONCLUSION}

Isolated cysticercosis of the abdominal wall is rare entity and the clinical diagnosis can be challenging as it can mimic other grave pathologies including acute abdomen due to its nonspecific clinical presentation as in our case. High resolution ultrasonography is proven to be a good modality for diagnosis and follow-up of soft tissue cysticercosis.

\section{REFERENCES}

1. Aghakhani N, Comoy J, Tadie M, Lacroix C, Bouree P. Isolated intramedullary cysticercosis. Casereport. Neurochirurgie 1998;44(2):127-131.
2. Hoberg EP. Taenia tapeworms: their biology, evolutionand socioeconomic significance. Microb Infect 2002;4(8):859-866.

3. McAdam AJ, Sharpe AH. Infectious diseases. In:Kumar V, Abbas AK, Nelson F, eds. Robbinsand Cotran pathologic basis of disease. Philadelphia:Elsevier Saunders; 2005.p.343-414.

4. Neafie RC, Marty AM, Johnson LK. Taeniasis and cysticercosis. In: Meyers WM, Neafie RC,Marty AM, Wear DJ, eds. Pathology of infectious diseases. Washington DC: AFIP;2000.p.117-136.

5. Kazanjian PH, Mattia AR. Case 26-1994: A 20-yearoldPhilippine woman with a soft-tissue mass inthe forearm. $N$ EnglJMed 1994;330(26):1887-1893.

6. Cysticercosis: an emerging parasitic disease.Am Fam Physician. 2007;76(1):91-96

7. Venkataraman S, Vijayan GP. Uncommon manifestation of human cysticercosis with muscular pseudohypertrophy. Trop Geogr Med 1983;35(1):75-77.

8. Abdelwahab IF, Klein MJ, Hermann G, Quader MA. Solitary cysticercosis of the biceps brachii in a vegetarian: a rare and unusual pseudotumor. Skeletal Radiol 2003;32(7):424-428.

9. Vijayaraghavan SB.Sonographic appearances in cysticercosis.J Ultrasound Med 2004;23(3):423-427.

10. Mittal A, Das D, Iyer N, Nagaraj J, Gupta M. Masseter cysticercosis- a rare case diagnosed on ultrasound. Dentomaxillofac Radiol 2008;37(2):113-116. 